\title{
Usability testing on government agencies web portal: a study on Ministry of Education Malaysia (MOE) web portal
}

\begin{abstract}
Web portals are emerging as a key component for government agencies in Malaysia to served public citizen in providing information and services in a more convenient, prompt, and secured platform. Usability of the web portal is one of the most important attributes for web portal quality because it generates persuasive factor to attract and satisfy users. This study evaluates the usability of the Ministry of Education Malaysia (MOE) web portal by conducting the usability testing in order to measure the usability level of the web portal and provides the relevant usability enhancement bas It also focused on evaluation of seven (7) usability attributes adopted from ISO/IEC 25010 and Website Analysis and Measurement Inventory (WAMMI) which are effectiveness, efficiency, learnability, controllability, attractiveness, helpfulness and satisfaction. The evaluation is limited to frequent tasks that ordinarily performed by the users on the MOE web portal which gathered from pilot study. Two stages of usability testing was conducted in this study which are pre-usability testing to evaluate the usability of the current MOE web portal and post-usability testing on the enhanced version of the MOE web portal. The pre-usability testing result showed that the usability of the current MOE web portal was at a moderate level for all the usability attributes and the post-usability testing result showed that the usability score improved significantly for all the usability attributes. This study proved that the usability testing method is one of the useful methods in enhancing web portal usability.
\end{abstract}

Keyword: Usability; Usability testing; Web portal 